\title{
A MAC Protocols using Networks
}

\author{
V. Ganesh Babu \\ Research Scholar \\ Vinayaka Missions University \\ Salem, Tamil Nadu
}

\author{
Dr. T. Senthil Kumar \\ Anna University-Tiruchirappali \\ Tiruchirappali, Tamil Nadu
}

\begin{abstract}
Wireless sensor networks are appealing to researchers due to their wide range of application in several fields such as military settings, critical infrastructure protection, target detection and tracking, environmental monitoring, industrial process monitoring etc. Communication among wireless sensor nodes is usually achieved by means of a unique channel. It is the characteristic of this channel that only a single node can transmit a message at any given time. Therefore, shared access of the channel requires the establishment of a MAC protocol among the sensor nodes. The objective of the MAC protocol is to regulate access to the shared wireless medium. In this paper, I first outline the sensor network properties that are crucial for the design of MAC layer protocols. Then, describe several MAC protocols proposed for sensor networks emphasizing their strengths and weaknesses. Finally, I point out open research issues on MAC layer design.
\end{abstract}

\section{Keywords}

MAC Protocols, Sensor Networks, Survey

\section{INTRODUCTION}

Wireless Sensor Networks are typically composed of a large number of low-cost, lowpower, multifunctional wireless devices deployed over a geographical area in an ad hoc fashion and without careful planning. Individually, sensing devices are resource constrained and therefore are only capable of a limited amount of processing and communication. It is the coordinated effort of these sensing devices, however, that bears promise for a significant impact on a wide range of applications in several fields, including science and engineering, military settings, critical infrastructure protection, and environmental monitoring . Harnessing the potential benefits of WSNs requires a high-level of self-organization and coordination among the sensors to perform the tasks required to support the underlying application. At the heart of this collaborative effort to achieve communications is the need for the wireless sensor nodes to self-organize into a multihop wireless network. Consequently, the design of efficient communications and network protocols for WSNs becomes crucial for wireless sensor nodes to carry out successfully the mission for which they are deployed. The establishment of a multihop wireless network infrastructure for data transfer requires the establishment of communication links between neighboring sensor nodes. Unlike communication over a guided medium in wired networks, however, communication in wireless networks is achieved in the form of electromagnetic signal transmission through the air. This common transmission medium must therefore be shared by all sensor network nodes in a fair manner. To achieve this goal, a medium access control protocol must be utilized. The choice of the medium access control protocol is the major determining factor in WSN performance. A number of access control protocols have been proposed for WSNs. The objective of this chapter is to discuss the fundamental design issues of medium access control for WSN methods and to provide an overview of these protocols. 


\section{MAC LAYER BACK GROUND}

Communication among wireless sensor nodes is usually achieved by means of a unique channel. It is the characteristic of this channel that only a single node can transmit a message at any given time. Therefore, shared access of the channel requires the establishment of a MAC protocol among the sensor nodes. The objective of the MAC protocol is to regulate access to the shared wireless medium such that the performance requirements of the underlying application are satisfied [1-4]. From the perspective of the Open Systems Interconnection (OSI) Reference Model (OSIRM), the MAC protocol functionalities are provided by the lower sublayer of the data link layer (DLL). The higher sublayer of the DLL is referred as the logical link control (LLC) layer. The subdivision of the data link layer into two sublayers is necessary to accommodate the logic required to manage access to a shared access communications medium. Furthermore, the presence of the LLC sublayer allows support for several MAC options, depending on the structure and topology of the network, the characteristics of the communication channel, and the quality of service requirements of the supported application. Figure 1.1 depicts the OSI reference model and the logical architecture of the DLL for shared medium access in wireless networks. The physical layer (PHY) typically includes a specification of the transmission medium and the topology of the network. It defines the procedures and functions that must be performed by the physical device and the communications interface to achieve bit transmission and reception. It also coordinates the various functions necessary to transmit a stream of bits over the wireless communication medium. The major services provided by the physical layer typically include the encoding and decoding of signals, preamble generation and removal to achieve synchronization, and the transmission and reception of bits. The MAC sublayer resides directly above the physical layer. It supports the following basic functions:

- The assembly of data into a frame for transmission by appending a header field containing addressing information and a trailer field for error detection

- The disassembly of a received frame to extract addressing and error control information to perform address recognition and error detection and recovery

- The regulation of access to the shared transmission medium in a way commensurate with the performance requirements of the supported application.

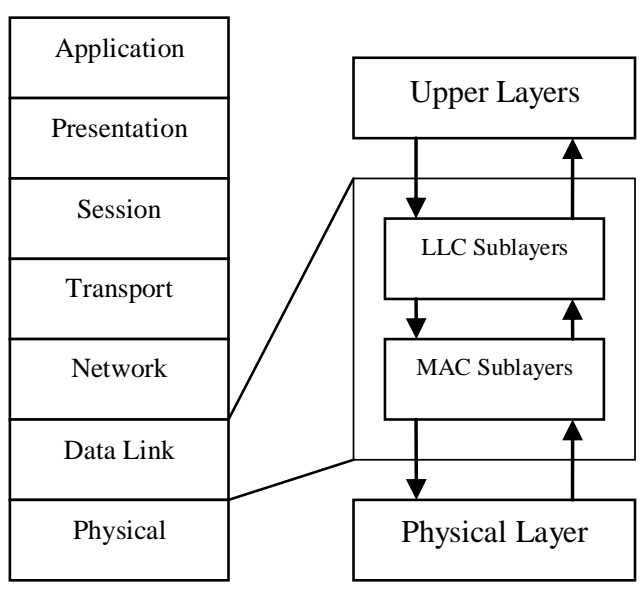

Figure 1.1

\section{Open systems interconnection reference model and data link layer architecture}

The LLC sublayer of the DDL provides a direct interface to the upper layer protocols. Its main purpose is to shield the upper layer protocols from the characteristics of the underlying physical network, thereby providing interoperability across different types of networks. The use of the LLC sublayer, however, has been very limited, as interoperability is typically achieved by other network layer protocols.

However, the medium access decision within a dense network composed of nodes with low duty-cycles is a hard problem that must be solved in an energy-efficient manner. Having these in mind, Section III emphasizes the peculiar 
features of sensor networks including reasons of potential energy wastes at medium access communication. Then, Section IV gives brief definitions for the key MAC protocols proposed for sensor networks listing their advantages and disadvantages. Moreover, the protocols that propose the integration of MAC layer with other layers also investigated in Section IV. Finally, Section V concludes the survey on MAC protocols with a comparison of investigated protocols and provides a future direction to researchers for open issues that have not been studied thoroughly

\section{MAC LAYER PROPERTIES}

Maximizing the network lifetime is a common objective of sensor network research, since sensor nodes are assumed to be disposed when they are out of battery. Under these circumstances, the proposed MAC protocol must be energy efficient by reducing the potential energy wastes presented in Section III.A. MAC Performance Matrices in III B ,Types of communication patterns that are observed in sensor network applications should be investigated since these patterns are used to extract the behavior of the sensor network traffic that has to be handled by a given MAC protocol. Categorization of the possible communication patterns are outlined in Section III.C. Afterwards, the properties that must be possessed by a MAC protocol to suit a sensor network environment are presented in Section III.D.

\section{A. Major Sources of Energy Wastes}

Major sources of energy waste in wireless sensor network are basically of four types .

\section{1) Collision:}

The first one is the collision. When transmitted packet is corrupted due to interference, it has to be discarded and the follow on retransmissions increase energy consumption. Collision increases latency also.

\section{2) Overhearing:}

The second is overhearing, meaning that a node picks up packets that are destined to other nodes.

\section{3) Packet Overhead:}

The third source is control packet overhead. Sending and receiving control packets consumes energy too and less useful data packets can be transmitted.

\section{4) Idle listening:}

The last major source of inefficiency is idle listening i.e., listening to receive possible traffic that is not sent. This is especially true in many sensor network applications. If nothing is sensed, the sensor node will be in idle state for most of the time. The main goal of any MAC protocol for sensor network is to minimize the energy waste due to idle listening, overhearing and collision.

\section{B. MAC Performance Matrices}

In order to evaluate and compare the performance of energy conscious MAC protocols, the following matrices are being used by the research community.

\section{1) Energy Consumption per bit: -}

The energy efficiency of the sensor nodes can be defined as the total energy consumed / total bits transmitted. The unit of energy efficiency is joules/bit. The lesser the number, the better is the efficiency of a protocol in transmitting the information in the network. This performance matrices gets affected by all the major sources of energy waste in wireless sensor network such as idle listening, collisions, control packet overhead and overhearing.

\section{2) Average Delivery Ratio: -}

The average packet delivery ratio is the number of packets received to the number of packets sent averaged over all the nodes.

\section{3) Average Packet Latency: -}

The average packet latency is the average time taken by the packets to reach to the sink node.

\section{4) Network Throughput:-}

The network throughput is defined as the total number of packets delivered at the sink node per time unit. 


\section{Communication Patterns}

Kulkarni et al. defines three types of communication patterns in wireless sensor networks [5]: broadcast, convergecast, and local gossip. Broadcast type of communication pattern is generally used by a base station (sink) to transmit some information to all sensor nodes of the network. Broadcasted information may include queries of sensor query-processing architectures, program updates for sensor nodes, control packets for the whole system. The broadcast type communication pattern should not be confused with broadcast type packet. For the former, all nodes of the network are intended receivers whereas for the latter the intended receivers are the nodes within the communication range of the transmitting node. In some scenarios, the sensors that detect an intruder communicate with each other locally. This kind of communication pattern is called local gossip, where a sensor sends a message to its neighboring nodes within a range. The sensors that detect the intruder, then, need to send what they perceive to the information center. That communication pattern is called convergecast, where a group of sensors communicate to a specific sensor. The destination node could be a clusterhead, data fusion center, base station. In protocols that include clustering, clusterheads communicate with their members and thus the intended receivers may not be all neighbors of the clusterhead, but just a subset of the neighbors. To serve for such scenarios, we define a fourth type of communication pattern, multicast, where a sensor sends a message to a specific subset of sensors.

\section{Properties of a Well-defined MAC Protocol}

To design a good MAC protocol for the wireless sensor networks, the following attributes must be considered [6]. The first attribute is the energy efficiency. We have to define energy efficient protocols in order to prolong the network lifetime. Other important attributes are scalability and adaptability to changes. Changes in network size, node density and topology should be handled rapidly and effectively for a successful adaptation. Some of the reasons behind these network property changes are limited node lifetime, addition of new nodes to the network and varying interference which may alter the connectivity and hence the network topology. A good MAC protocol should gracefully accommodate such network changes. Other typical important attributes such as latency, throughput and bandwidth utilization may be secondary in sensor networks. Contrary to other wireless networks, fairness among sensor nodes is not usually a design goal, since all sensor nodes share a common task.

\section{PROPOSED MAC LAYER ROTOCOLS}

In this section, a wide range of MAC protocols defined for sensor networks are described briefly by stating the essential behavior of the protocols wherever possible. Moreover, the advantages and disadvantages of these protocols are presented.

\section{A. IEEE 802.11}

The IEEE 802.11[21] is a well known contention based medium access control protocol which uses carrier sensing and randomized backoffs to avoid collisions of the data packets. The Power Save Mode(PSM) of the IEEE 802.11 protocol reduces the idle listening by periodically entering into the sleep state. This PSM mode is for the single-hop network where the time synchronization is simple and may not be suitable for multi-hop networks because of the problems in clock synchronization, neighbour discovery and network partitioning.

\section{B. PAMAS: Power Aware Multi- Access Signaling}

PAMAS: Power Aware Multi-Access is one of the earliest contention based MAC protocol designed with energy efficiency as the main objective. In this protocol nodes which are not transmitting or receiving are turned "OFF" in order to conserve energy. This protocol uses two separate channels for the data and control packets. It requires the use of two radios in the different frequency bands at each sensor node 
leading to the increase in the sensors cost, size and design complexity. Moreover, there is significant power consumption because of excessive switching between sleep and wakeup states.

\section{Sensor-MAC (S-MAC)page}

The sensor-MAC (S-MAC) protocol [6] is designed explicitly to reduce energy waste caused by collision, idle listening, control overhead, and overhearing. The goal is to increase energy efficiency while achieving a high level of stability and scalability. In exchange, the protocol incurs some performance reduction in per-hop fairness, and latency S-MAC uses multiple techniques to reduce energy consumption, control overhead, and latency, in order to improve application-level performance. In the following we provide an overview of the S-MAC-layer protocol and discuss the techniques it proposes to achieve energy efficiency while keeping latency low.

Schedule exchanges are accomplished by periodical SYNC packet broadcasts to immediate neighbors. The period for each node to send a SYNC packet is called the synchronization period. Figure 1.2 represents a sample senderreceiver communication. Collision avoidance is achieved by a carrier sense, which is represented as CS in the figure. Furthermore, RTS/CTS packet exchanges are used for unicast type data packets.

An important feature of S-MAC is the concept of message-passing where long messages are divided into frames and sent in a burst. With this technique, one may achieve energy savings by minimizing communication overhead at the expense of unfairness in medium access. Periodic sleep may result in high latency especially for multi-hop routing algorithms, since all immediate nodes have their own sleep schedules. The latency caused by periodic sleeping is called sleep delay in [6]. Adaptive listening technique is proposed to improve the sleep delay, and thus the overall latency. In that technique, the node who overhears its neighbor's transmissions wakes up for a short time at the end of the transmission. Hence, if the node is the next-hop node, its neighbor could pass data immediately. The end of the transmissions is known by the duration field of RTS/CTS packets.

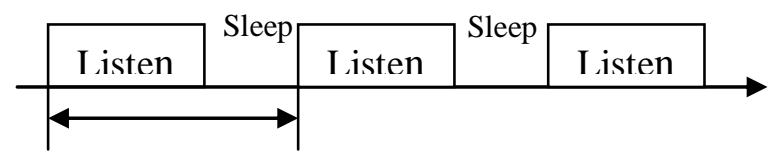

Frame

FIGURE 1.2

\section{S-MAC PERIOD LISTEN AND SLEEP MODES OF} OPERATIONS

Advantages: The energy waste caused by idle listening is reduced by sleep schedules. In addition to its implementation simplicity, time synchronization overhead may be prevented with sleep schedule announcements

Disadvantages: Broadcast data packets do not use RTS/CTS which increases collision probability. Adaptive listening incurs overhearing or idle listening if the packet is not destined to the listening node. Sleep and listen periods are predefined and constant, which decreases the efficiency of the algorithm under variable traffic load.

\section{Timeout T-MAC}

Timeout T-MAC [3] is the protocol based on the $\mathrm{S}-\mathrm{MAC}$ protocol in which the Active period is preempted and the sensor goes to the sleep period if no activation event has occurred for a time 'Ta' as shown in Fig.1.3. The event can be reception of data, start of listen/sleep frame time etc. The time ' $\mathrm{Ta}$ ' is the minimal amount of idle listening per frame. The interval $\mathrm{Ta}>\mathrm{Tci}+\mathrm{Trt}$ $+\mathrm{Tta}+\mathrm{Tct}$ where Tci is the length of the contention interval, Trt is the length of an RTS packet, Tta is the turn-around time (time between the end of the RTS packet and the beginning of the CTS packet) and Tct is the length of the CTS packet. The energy consumption in the Timeout TMAC protocol is less than the Sensor S-MAC protocol. But the Timeout T-MAC protocol has high latency as compared to the S-MAC protocol. Figure 1.3: Basic T-MAC Scheme 


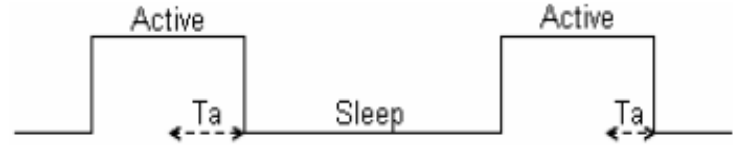

FIGURE 1.3: BASIC T-MAC SCHEME

\section{E. WiseMAC}

Spatial TDMA and CSMA with Preamble Sampling protocol is proposed in [7] where all sensor nodes are defined to have two communication channels. Data channel is accessed with TDMA method, whereas the control channel is accessed with CSMA method. Enz et al. proposed WiseMAC [8] protocol which is similar to Hoiydi et al.'s work [7] but requires only a single-channel. WiseMAC protocol uses non-persistent CSMA (np-CSMA) with preamble sampling as in [7] to decrease idle listening. In the preamble sampling technique, a preamble precedes each data packet for alerting the receiving node. All nodes in a network sample the medium with a common period, but their relative schedule offsets are independent. If a node finds the medium busy after it wakes up and samples the medium, it continues to listen until it receives a data packet or the medium becomes idle again. The size of the preamble is initially set to be equal to the sampling period.

However, the receiver may not be ready at the end of the preamble, due to reasons like interference, which causes the possibility of overemitting type energy waste. Moreover, overemitting is increased with the length of the preamble and the data packet, since no handshake is done with the intended receiver. To reduce the power consumption incurred by the predetermined fixed-length preamble, WiseMAC offers a method to dynamically determine the length of the preamble. That method uses the knowledge of the sleep schedules of the transmitter node's direct neighbors. The nodes learn and refresh their neighbor's sleep schedule during every data exchange as part of the acknowledgement message. In that way, every node keeps a table of sleep schedules of its neighbors. Based on neighbors' sleep schedule table, Wise MAC schedules transmissions so that the destination node's sampling time corresponds to the middle of the sender's preamble. To decrease the possibility of collisions caused by that specific start time of wake-up preamble, a random wake-up preamble is advised. Another parameter affecting the choice of the wake-up preamble length is the potential clock drift between the source and the destination. A lower bound for the preamble length is calculated as the minimum of destination's sampling period, Tw, and the potential clock drift with the destination which is a multiple of the time since the last ACK packet arrival. Considering this lower bound, a preamble length, Tp, is chosen

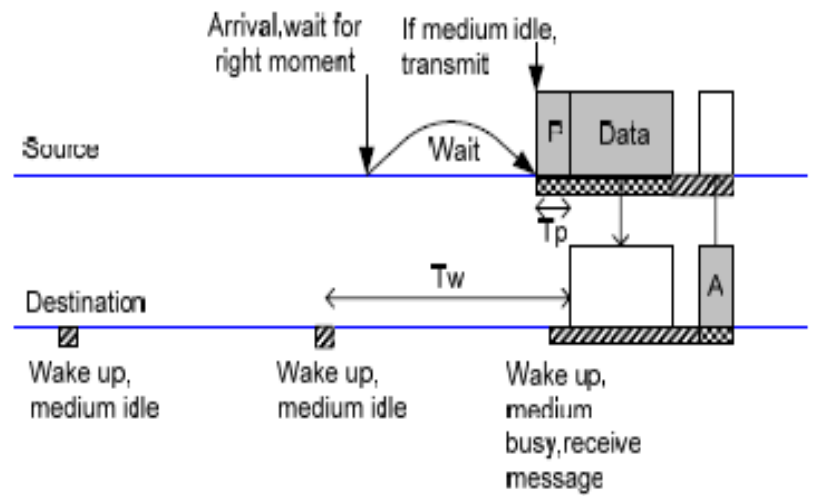

randomly. Figure 1.4 presents the Wise MAC concept.

QRX 因 TX P: Preamble A: Acknowledge

\section{FIGURE 1.4 WISE MAC CONCEPTS}

Advantages: The simulation results show that WiseMAC performs better than one of the SMAC variants [4]. Besides, its dynamic preamble length adjustment results in better performance under variable traffic conditions. In addition, clock drifts are handled in the protocol definition which mitigates the external time synchronization requirement. 
Disadvantages: Main drawback of WiseMAC is that decentralized sleep-listen scheduling results in different sleep and wake-up times for each neighbor of a node. This is especially an important problem for broadcast type of communication, since broadcasted packet will be buffered for neighbors in sleep mode and delivered many times as each neighbor wakes up. However, this redundant transmission will result in higher latency and power consumption. In addition, the hidden terminal problem comes along with WiseMAC model as in the Spatial TDMA and CSMA with Preamble Sampling algorithm. That is because WiseMAC is also based on non-persistent CSMA. This problem will result in collisions when one node starts to transmit the preamble to a node that is already receiving another node's transmission where the preamble sender is not within the range.

\section{F. Traffic-Adaptive MAC Protocol (TRAMA)}

TRAMA [9] is a TDMA-based algorithm and proposed to increase the utilization of classical TDMA in an energy efficient manner. It is similar to Node Activation Multiple Access (NAMA) [10], where for each time slot a distributed election algorithm is used to select one transmitter within two-hop neighborhood. This kind of election eliminates the hidden terminal problem and hence, ensures all nodes in the one-hop neighborhood of the transmitter will receive data without any collision. However, NAMA is not energyefficient, and incurs overhearing. Time is divided into random-access and scheduled-access (transmission) periods. Random-access period is used to establish twohop topology information where channel access is contention-based. A basic assumption is that, by the information passed by the application layer, MAC layer can calculate the transmission duration needed which is denoted as SCHEDULE_INTERVAL. Then at time $t$, the node calculates the number of slots for which it will have the highest priority among two-hop neighbors within the period [t,t+ SCHEDULE_INTERVAL]. The node announces the slots it will use as well as the intended receivers for these slots with a schedule packet. Additionally, the node announces the slots for which it has the highest priority but will not be used. The schedule packet indicates the intended receivers using a bitmap whose length is equal to the number of its neighbors. Bits correspond to one-hop neighbors ordered by their identities. Since the receivers of those messages have the exact list and identities of the on hop neighbors, they find out the intended receiver. When the vacant slots are announced, potential senders are evaluated for re-use of those slots. Priority of a node on a slot is calculated with a hash function of node's and slot's identities. Analytical models for the delay performances of TRAMA and NAMA protocols are also presented and supported by simulations [9]. Delays are found to be higher compared to contention-based protocols due to higher percentage of sleep times.

Advantages: Higher percentage of sleep time and less collision probability is achieved compared to CSMA based protocols. Since intended receivers are indicated with a bitmap, less communication is performed for multicast and broadcast type of communication patterns compared other protocols.

Disadvantages: Transmission slots are set to be seven times longer than the random access period [5]. However, all nodes are defined to be either in receive or transmit states during the random access period for schedule exchanges. This means that without considering the transmissions and receptions, the duty cycle is at least $12.5 \%$, which is a considerably high value. For a time slot, every node calculates each of its two-hop neighbors' priorities on that slot. In addition, this calculation is repeated for each time slot, since the parameters of the calculation change with time.

\section{G. SIFT}

Sift [11] is a MAC protocol proposed for event-driven sensor network environments. The motivation behind Sift is that when an event is sensed, the first $\mathrm{R}$ of $\mathrm{N}$ potential reports is the most crucial part of messaging and has to be relayed with low latency. Jamieson et al. use a non-uniform probability distribution function of picking a slot within the slotted contention 
window. If no node starts to transmit in the first slot of the window, then each node increases its transmission probability exponentially for the next slot assuming that the number of competing nodes is small. In [11], Sift is compared with 802.11 MAC protocol and it is showed that Sift decreases latency considerably when there are many nodes trying to send a report. Since Sift is a method for contention slot assignment algorithm, it is proposed to co-exist with other MAC protocols like SMAC. Based on the same idea, CSMA/p* is proposed in [12] where $\mathrm{p}^{*}$ is a non-uniform probability distribution that optimally minimizes latency. However, Tay et al. state that Sift has a distribution approximate to CSMA/p*.

Advantages: Very low latency is achieved with many traffic sources. Energy consumption is traded off for latency as indicated below. However, when the latency is an important parameter of the system, slightly increased energy consumption must be accepted. It could be tuned to incur less energy consumption. The high energy consumption is a result of the arguments indicated below.

Disadvantages: One of the main drawbacks is increased idle listening caused by listening to all slots before sending. The second drawback is increased overhearing. When there is an ongoing transmission, nodes must listen till the end in order to contend for the next transmission which causes overhearing. Besides, system-wide time synchronization is needed for slotted contention windows. That is why, the implementation complexity of Sift would be increased for the protocols not utilizing time synchronization.

\section{H. DMAC}

Convergecast is the mostly observed communication pattern within sensor networks. These unidirectional paths from possible sources to the sink could be represented as data gathering trees. The principal aim of DMAC [13] is to achieve very low latency, but still to be energy efficient. DMAC could be summarized as an improved Slotted Aloha algorithm where slots are assigned to the sets of nodes based on a data gathering tree as shown in Figure 1.5. Hence, during the receive period of a node, all of its child nodes has transmit periods and contend for the medium. Low latency is achieved by assigning subsequent slots to the nodes that are successive in the data transmission path.

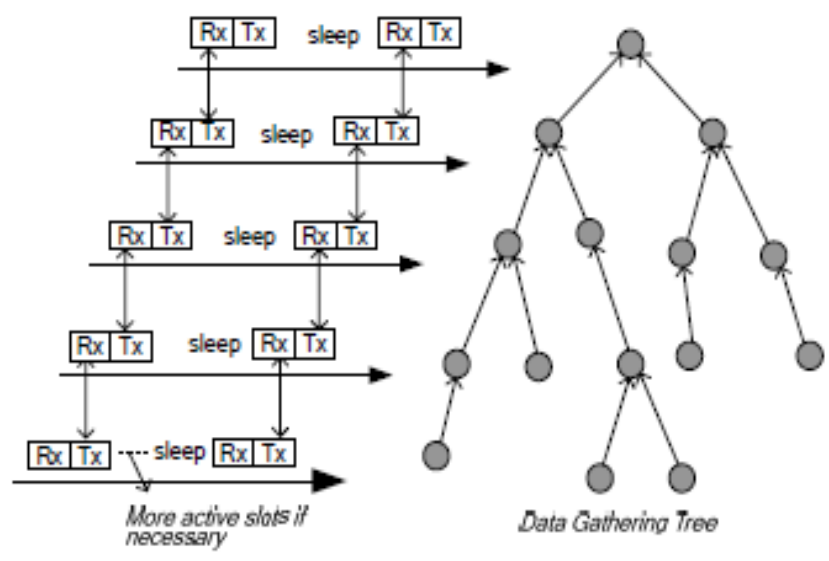

\section{FIG. 1.5 A DATA GATHERING TREE AND ITS DMAC IMPLEMENTATION}

Advantages: DMAC achieves very good latency compared to other sleep/listen period assignment methods. The latency of the network is crucial for certain scenarios, in which DMAC could be a strong candidate.

Disadvantages: Collision avoidance methods are not utilized, hence when a number of nodes that has the same schedule (same level in the tree) try to send to the same node, collisions will occur. This is a possible scenario in eventtriggered sensor networks. Besides, the data transmission paths may not be known in advance, which precludes the formation of the data gathering tree.

\section{Timeout-MAC (T-MAC) / Dynamic Sensor-MAC(DSMAC)}

Static sleep-listen periods of S-MAC result in high latency and lower throughput as indicated earlier. Timeout-MAC (T-MAC) [14] is proposed to enhance the poor results of S-MAC protocol under variable traffic load. In T-MAC, listen period ends when no activation event has occurred for a time threshold TA. The decision 
for TA is presented along with some solutions to the early sleeping problem defined in [14]. Variable load in sensor networks are expected, since the nodes that are closer to the sink must relay more traffic. Although T-MAC gives better results under these variable loads, the synchronization of the listen periods within virtual clusters is broken. This is one of the reasons for the early sleeping problem. Dynamic Sensor-MAC (DSMAC) [15] adds dynamic duty cycle feature to S-MAC. The aim is to decrease the latency for delay-sensitive applications. Within the SYNC period, all nodes share their one-hop latency values (time between the reception of a packet into the queue and its transmission). All nodes start with the same duty cycle. Figure 1.6 conceptually depicts DSMAC duty cycle doubling. When a receiver node notices that average one-hop latency value is high, it decides to shorten its sleep time and announces it within SYNC period. Accordingly, after a sender node receives this sleep period decrement signal, it checks its queue for packets destined to that receiver node. If there is one, it decides to double its duty cycle when its battery level is above a specified threshold.

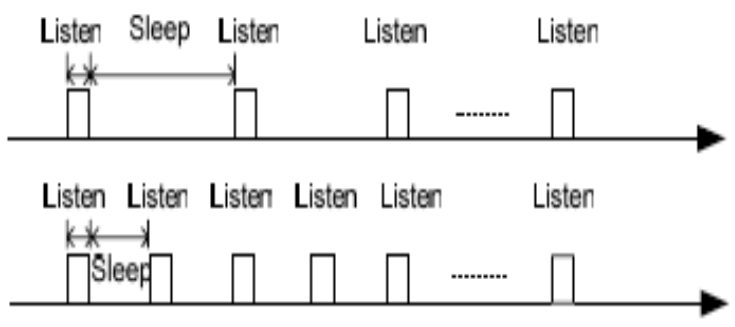

FIG 1.6. DSMAC DUTY CYCLE DOUBLING [15]

The duty cycle is doubled so that the schedules of the neighbors will not be affected. The latency observed with DSMAC is better than the one observed with S-MAC. Moreover, it is also shown to have better average power consumption per packet.

\section{$J$. Integration of MAC with Other Layers}

Limited research has been carried out to integrate different network layers to one layer or to benefit from the cross-layer interactions between routing and MAC layers for sensor networks. One such research is done by Safwat et al. who proposed two routing algorithms that favor the information about successful/unsuccessful CTS or ACK reception [16].Cui et al. have research in that area with the objectives of MAC/Physical layer integration and Routing/ MAC/Physical layer integration [17]. They propose a variable length TDMA scheme where the slot length is assigned according to some criteria for the optimum energy consumption in the network. Among these criteria, the most crucial ones are information about the traffic generated by each node and distances between each node pair. Based on these values, they formulate a Linear Programming (LP) problem where the decision variables are normalized time slot lengths between nodes. They solve this LP problem using an LP solver which returns the optimum number of time slots for each node pairs as well as the related routing decisions for the system. The proposed solution could be quite beneficial for scenarios where the required data could be prepared. However, it is generally hard to have the node distance information and the traffic generated by the nodes. Besides, LP solver could only be run on a powerful node. However, the dynamic behaviors of sensor networks will require online decisions which are very costly to calculate and hard to adapt to an existing system. Multihop Infrastructure Network Architecture (MINA) is another work for integrating MAC and routing protocols b[18]. Ding et al. propose a layered multi-hop network architecture where the network nodes with the same hopcount to the base station are grouped into the same layer. Channel access is a TDMA-based MAC protocol combined with CDMA or FDMA. The superframe is composed of a control packet, a beacon frame and a data transmission frame. Beacon and data frames are time slotted. In the clustered network architecture, all members of a cluster submit their transmission requests in beacon slots. Accordingly, the cluster-head announces the schedule of the data frame. The routing protocol is a simple multi-hop protocol where each node has a forwarder node at one nearer layer to the base station. The forwarding node is 
chosen from candidates based on the residual energies. Ding et al. then formulate the channel allocation problem as an NP-complete problem and propose a sub-optimal solution. Moreover, the transmission range of sensor nodes is a decision variable, since it affects the layering of the network (hop-counts change). Simulations are run to find a good range of values for a specific scenario. The proposed system in [18] is a well-defined MAC/Routing system. However, the tuning of the range parameter is an important task which should be determined at the system initialization. In addition, all node-to-sink paths are defined at the startup and are defined to be static, since channel frequency assignments of nodes are done at the startup accordingly. This makes the system intolerant to failures. Geographic Random Forwarding (GeRaF) is actually proposed as a routing protocol, but the underlying MAC algorithm is also defined in the work which is based on CSMA/CA [19]. That gives us not integrated but a complete solution for a sensor network's communication layers. The difficulty of the system proposed is its need for additional radio, which is used for busy tone announcement. Rugin et al. [20] and Zorzi et al. [15] improved GeRaF reducing it to a onechannel system. However, sensor nodes' and their neighbors' location information are needed for those protocols. Besides, the forwarding node is chosen among nodes that are awake at the time of the transmission request. That may result in more power consuming routing, and an increase in latency.

\section{OPEN ISSUES AND CONCLUSIONS}

Table I represents a comparison of MAC protocols investigated. Time Synchronization needed column indicates whether the protocol assumes that the time synchronization is achieved externally.

Adaptivity to Changes means ability to handle topology changes. The two S-MAC variants, namely, T-MAC and DSMAC, have the same features with S-MAC given in Table I. The cross-layer protocols include additional layers other than the MAC layer, and are not considered in this comparison.

Table I Comparion of MAC Protocols

\begin{tabular}{|c|c|c|c|c|}
\hline & $\begin{array}{c}\text { Time } \\
\text { Synch. } \\
\text { Needed }\end{array}$ & $\begin{array}{c}\text { Com } \\
\text { m. } \\
\text { Patter } \\
\mathbf{n} \\
\text { Suppo } \\
\text { rt }\end{array}$ & Type & $\begin{array}{c}\text { Adap- } \\
\text { tivity } \\
\text { to } \\
\text { Chan- } \\
\text { ges }\end{array}$ \\
\hline $\begin{array}{c}\text { S-MAC/ } \\
\text { T-MAC/ } \\
\text { DSMAC }\end{array}$ & No & All & CSMA & Good \\
\hline WiseMAC & No & All & $\begin{array}{c}\text { ns- } \\
\text { CSMA }\end{array}$ & Good \\
\hline TRAMA & Yes & All & $\begin{array}{c}\text { TDMA/ } \\
\text { CSMA }\end{array}$ & Good \\
\hline SIFT & No & All & $\begin{array}{c}\text { CSMA/ } \\
\text { CA }\end{array}$ & Good \\
\hline DMAC & Yes & $\begin{array}{c}\text { Conve } \\
\text { r- } \\
\text { gecast }\end{array}$ & $\begin{array}{c}\text { TDMA/ } \\
\text { Slotted } \\
\text { Aloha }\end{array}$ & Weak \\
\hline
\end{tabular}

Although there are various MAC layer protocols proposed for sensor networks, there is no protocol accepted as a standard. One of the reasons behind this is the MAC protocol choice will, in general, be application-dependent, which means that there will not be one standard MAC for sensor networks. Another reason is the lack of standardization at lower layers (physical layer) and the (physical) sensor hardware. TDMA has a natural advantage of collision-free medium access. However, it includes clock drift problems and decreased throughput at low traffic loads due to idle slots. The difficulty with TDMA systems are the synchronization of the nodes and adaptation to topology changes where these changes are caused by insertion of new nodes, exhaustion of battery capacities, broken links because of interference, sleep schedules of relay nodes, scheduling caused by clustering algorithms. The slot assignments, therefore, should be done regarding such possibilities. However, it is not easy to change the slot assignment within a decentralized environment for traditional TDMA, since all nodes must agree on the slot assignments. In parallel with the common networking lore, CSMA methods have 
a lower delay and promising throughput potential at lower traffic loads, which generally happens to be the case in wireless sensor networks. However, additional collision avoidance or collision detection methods should be employed to handle the collision possibilities. FDMA is another scheme that offers a collision-free medium. Though, it brings an additional circuitry requirement to dynamically communicate with different radio channels. This increases the cost of the sensor nodes, which is contrary to the objective of the sensor network systems. CDMA also offers collision-free medium, but its high computational requirement is a major obstacle for less energy consumption objective of the sensor networks. In pursuit of low computational cost requirements of wireless CDMA sensor networks, there has been limited effort to investigate source and modulation schemes, particular signature waveforms, designing simple receiver models, and other signal synchronization problems. If it is shown that the high computational complexity of CDMA could be traded with its collision avoidance feature, CDMA protocols could also be considered as candidate solutions for sensor networks. Lack of comparison of TDMA, CSMA or other medium access protocols in a common framework is a crucial deficiency of the literature. Common wireless networking experience also suggests that link-level performance alone may provide misleading conclusions about the system performance. Similar conclusion can be drawn for upper layers as well. Hence, the more layers contributing to the decision, the more efficient the system can be. For instance, the routing path could be chosen depending on the collision information from the medium access layer. Moreover, layering of the network protocols creates overheads for each layer which causes more energy consumption for each packet. Therefore, integration of the layers is also a promising research area which has to be studied more extensively.

\section{REFERENCES}

[1] V. Bharghavan, A. Demers, S. Shenker, L. Zhang, "MACAW : A Media Access
Protocol for Wireless LANs", proceedings of the ACM Sigcomm,, Portland, Oregon, 1994, PP 212-225

[2] T.V. Dam, K. Langendoen, "An Adaptive Energy-Efficient MAC Protocol for Wireless Sensor Networks", proceedings of the $1^{\text {st }}$ ACM Conference on Embedded Networked Sensor Systems (SENSYS'03), Los Angeles, Nov 2003.

[3] J. Ding, K. Sivalingam, R. Kashyapa, L.J. Chuan, "A Multi-layered Architecture and Protocols for Large-Scale Wireless Sensor Networks", proceedings of the IEEE $58^{\text {th }}$ Vehicular Technology Conference (VTC'03), Oct. 2003, Vol.3, PP. 1443.

[4] P. Karn, "MACA : A New Channel Access Method for Packet Radio", proceedings of the APRL / CRRL Amateur Radio $9^{\text {th }}$ Computer Networking Conference, Sept. 1990, PP. 134-140.

[5] S.S. Kulkarni, "TDMA Services for Sensor Networks", proceedings of $24^{\text {th }}$ International Conference on Distributed Computing Systems Workshops, Pages : 604-609, 23-24 March 2004

[6] W.Ye, J. Heidemann, D Estrin, "Medium Access Control with Coordinated Adaptive Sleeping of Wireless Sensor Networks", IEEE / ACM Transactions on Networking, Volume : 12, Issur : 3, Pages ; 493 - 506, June 2004.

[7] A. El-Hoiydi, "Spatial TDMA and CSMA with preamble sampling for low power Adhoc wireless Sensor Networks", proceedings of ISCC 2002, Seventh International Symposium on Computers and Communications , Pages L 685 - 692, 1-4 July 2002.

[8] C.C. Enz, A. El-Hoiydi, J.D. Decotignie, V. Peiris, "Wisenet : An Ultralow Power Wireless Sensor Network Solution", IEEE Computer Volume : 37, Issue : 8, August 2004.

[9] V. Rajendran, K. Obraczka, J.J. GarcialunaAceves, "Energy - Efficient, Collision-free Medium Access Control for Wireless Sensor Networks", proceddings of ACM SENSYS 03, Pages : 181 - 192, Los Angeles, California, 5-7 November 2003. 
[10] L. Bao and J.J. Garcia-Luna-Aceves, "A New Approach to Channel Access Scheduling for Ad Hoc Networks", $7^{\text {th }}$ Annual International Conference on Mobile Computing and Networking, PP. 210-221, 2007.

[11] K. Jamieson, H. Balakrishnan and Y.C. Tay, "SIFT : A MAC Protocol for Event Driven Wireless Sensor Networks", MIT Laboratory for Computer Science, Tech. Rep. 894, May 2003.

[12] Y.C. Tay, K. Jamieson, H. Balakrishnan, "Collision-Minimizing CSMA and its applications to Wireless Sensor Networks", IEEE Journal on selected areas in Communications, Volume : 22 .

[13] G. Lu, B. Krishnamachari, C.S. Raghavendra, “ An Adaptive Energy Efficient and Low-latency MAC for Data gathering in Wireless Sensor Networks", proceedings of $18^{\text {th }}$ International Parallel and Distributed Proessing Symposium, Pages : 224, 26-30 April 2004.

[14] T.V. Dam and K. Langendoen, " An Adaptive Energy-Efficient MAC Protocol for Wireless Sensor Networks", the first ACM Conference on Embedded Networked Sensor Systems (SENSYS'03), Los Angeles, CA, USA, November 2003.

[15] P. Lin, C. Qiao and X. Wang, "Medium Access Control with a Dynamic Duty Cycle for Sensor Networls", IEEE Wireless communications and Networking Conference, Volume : 3, Pages : 1534 1539, 21-25 March 2004
[16] A. Safwat, H. Hassanein, H. Mouftah, "ECPS and E2LA : New Paradigms for Energy Efficiency in Wireless Adhoc and Sensor Networks", IEEE Global Telecommunications Conference, GLOBECOM'03, Volume : 6, Pages : 3547 - 3552, 1-5 December 2007

[17] S. Cui, R. Madan, A. J. Goldsmith and S. Lall, “ Joint Routing, MAC, and Link Layer Optimization in Sensor Networks with Energy Constraints", to appear at ICC'05, Korea, may 2005

[18] J. Ding, K. Sivalingam, R Kashyapa, L.J. Chuan, "A Multi-layered Architecture and Protocols for Large-scale Wireless Sensor Networks", IEEE $58^{\text {th }} \quad$ Vehicular Technology Conference, 2003, VTC.

[19] M. Zorzi, "A New Contention-based MAC Protocol for Geographic forwarding in Adhoc and Sensor Networks", IEEE International Conference on Communications, Pages : $3481-3485$, Vol 6

[20] R. Rugin, G. Mazzini, "A Simple and Efficient MAC Routing Integrated Algorithm for Sensor Network", IEEE International Conference on communications, Volume : 6, Pages : 34993503, 20-24 June 2004.

[21] Rajesh Yadav, Shirshu Varma, N Malaviya, "A Survey of MAC Protocols for Wireless Sensor Networks", UBICC Journal, Volume 4, Number 3, August 2009. 